\title{
PASSBANDS AND PHOTOMETRIC SYSTEMS
}

\author{
F. Rufener \\ Geneva Observatory \\ CH-1290 SAUVERNY \\ Switzerland
}

\begin{abstract}
Synthetic photometry must be used to accurately define the passbands which characterize a photometric system. On the one hand, the colour indices of the stars must be coherent with their absolute spectrophotometry; on the other hand, direct comparisons of model atmospheres with observations must be carried out without resorting to arbitrary transformations. A systematic evaluation procedure could be applied to various photometric systems.
\end{abstract}

\section{INTRODUCTION}

The comment I propose to make on this subject is determined by the typical circumstance encountered, which I summarize as follows:

The passbands of a given system are described by their profiles in one of the first publications concerning the system. Insufficient coherence with the standard of the system precludes that they be directly used, for example, in the interpretation of synthetic photometry. Rather, a transformation (addition of a constant, or linear transformation) is required for comparing the computed magnitudes to the standard. We must bear in mind, however, that this is equivalent to multiplying the computed fluxes by a step function and using an exponent that is variable from one passband to another - which has little physical significance. It is as necessary as it is unfortunate that this method must already be used when one transforms into a known standard the observations made in a natural system. What are the remedies to this situation?

\section{CHARACTERIZING A PHOTOMETRIC SYSTEM}

Each photometric system should be characterized by a standard and by a coherent description of the passbands.

In other words, the magnitudes and indices of the standard stars should be comparable in a straightforward manner and with good precision to the values 
obtained by filtering their spectrophotometric data through the characteristic passbands, $\Phi_{i}(\lambda)$. This goal can be attained in several manners, the simplest probably being:

(1) Collect a sample of spectrophotometrically well known stars, i.e., which are well correlated with an absolute calibration, such as that of $\alpha$ Lyr. These stars should be distributed over the whole HR diagram, and should provide a large overlap with the standard stars of the photometric system.

(2) Provide, for each passband, an accurate spectral response, or its convolution with that of an appropriate detector: $\phi_{i}(\lambda)$.

(3) Apply a calibration procedure which defines the responses of the collector optics convolved by that of the detector if it is unique: $S(\lambda)$. During this calibration it is useful to determine at the same time the equalisation factors, $f_{i}$, which take into account the possible scaling errors in the functions $\phi_{i}(\lambda)$. In particular, this factor $f_{i}$ accounts for the changes in the fraction of incident light reflected off the air-glass surfaces of the filter, depending on its location either on the optical workbench or in the photometer.

(4) Obtain the overall characteristic passband function from the product

$$
\Phi_{i}(\lambda)=f_{i} \cdot S(\lambda) \cdot \phi_{i}(\lambda)
$$

\section{NATURAL AND STANDARD PHOTOMETRIC SYSTEMS}

Each natural system used for the acquisition of new observations must be very close to that which defines the standard.

This condition is extremely important if we wish to interpret the new observations by means of calibration of the standard system. A correlation by linear transformation of the natural magnitudes and indices, if necessary, would then have very little effect. Particularly, the extent of the spectral domain covered has to be maintained very similar. The responsibility of the observers is very important; they have to take great care during the realization of the natural systems. This constraint is all the more difficult to satisfy as the materials and instruments which define the passbands evolve, and as detectors are produced with varying characteristics. Today, this responsibility lies most often in the hands of collectively run observatories (such as ESO or AURA) where it is truly necessary that all precautions be taken and that there be on the site one responsible person known to all visiting astronomers.

4. SETTING UP THE STRATEGY:

APPLICATION TO GENEVA PHOTOMETRY

The collection of spectra obtained by Gunn \& Stryker (1983), G\&S hereafter, ap- 
pears to be well adapted as spectrophotometric reference for this task because of the following properties:

- Absolute calibration adjusted on $\alpha$ Lyr (Hayes \& Latham, 1975), extended by means of an elaborate transfer procedure to five subdwarf stars (Oke \& Gunn, 1983) as secondary standards.

- A variety of stars well representing the whole HR diagram and its different populations.

- Extensive spectrophotometric description ( $3130 \AA$ to $10800 \AA$ ). A small extension towards the UV would however be desirable. (We have made an extrapolation down to $3000 \AA$ ). The stars chosen by G\&S are only rarely standards of existing photometric systems, and not even all are well measured in each system. This shortcoming can be lifted in the future.

The comments of G\&S and of Oke \& Gunn (1983) draw attention to the difficulties underlying the transfer of the absolute calibration of $\alpha$ Lyr towards fainter stars. They also mention the perturbations due to cirrus clouds during the observations and the corrections carried out in such cases. If this catalogue closely fits our requirements for the computation we intend to perform, we must nevertheless accept the risk that it may not be as perfect yet as postulated.

To test the validity of the proposed method, we have applied it to Geneva photometry. The present overlap between the catalogue of Geneva photometry and that of G\&S contains 108 stars, discounting the subdwarfs which were used as secondary spectrophotometric standards and do not figure in the G\&S catalogue. Some stars have been eliminated because of their variability. The calibration procedure reflects that used by Rufener \& Maeder (1971), R\&M hereafter. We have chosen to express $\Phi_{i}(\lambda)$ in terms of a quantum response. Without dwelling on all the technical details, which will be presented elsewhere (Nicolet \& Rufener, in preparation), I will give here a summary.

The function $S(\lambda)$ is parameterised by means of piecewise cubic splines. Continuity is imposed as well as the equality of the slopes at the junction wavelengths, which are chosen close to the mean wavelength of each passband. The tangent is set to zero at the extremes of the sensitivity domain where $S(\lambda)=0$. The factors $f_{i}$ and the parameters of the function $S(\lambda)$ are optimized by minimizing the sum of the deviations in flux, expressed as photons per second. The mean adjustment which results for all passbands (7) over 101 stars takes into account the normalisation given by G\&S for the V-band.

The influence of low fluxes was reduced by weighting. The weight diminishes exponentially with the flux as soon as the magnitude for a given band exceeds 8.5. This precaution is necessary because the signal to noise ratio of the spectrophotometric data gets lower. Other reasons for weighting were also considered, but were finally seen to be of little or no consequence. This was the case for the very blue stars for which the extrapolation of the spectrophotometry to $3000 \AA$ could play a role; or in the case of the relatively higher importance of the $B$ and $V$ bands, which 
results from their being wider than the intermediate passbands $\left(U, B_{1}, B_{2}, V_{1}, G\right)$.

Encouraging results were obtained. The table gives, for each colour, the mean deviations in flux with their r.m.s. value calculated over 101 stars. The table also gives the mean differences in colours (indices) and their r.m.s. fluctuations.

\begin{tabular}{|l|c|c|c|c|c|c|c|}
\hline & $U$ & $B_{1}$ & & $B_{2}$ & $V_{1}$ & $V$ & $G$ \\
\hline $\begin{array}{l}\text { Mean of the deviations } \\
\text { in flux 1 over 101 } \\
\text { stars (measured- } \\
\text { calculated) } \\
\begin{array}{l}\sigma \text { over this mean } \\
\text { (r.m.s.) }\end{array}\end{array}$ & 0 & 0 & 0 & 0 & 0 & 0 & 0 \\
\hline $\begin{array}{l}\text { Mean of the deviations } \\
\text { of colours (indices) } \\
\text { in magnitudes } \\
\begin{array}{l}\sigma \text { over this mean } \\
\text { (r.m.s.) }\end{array}\end{array}$ & \pm .014 & \pm .011 & \pm .017 & \pm .006 & \pm .006 & \pm .014 & \pm .006 \\
\hline
\end{tabular}

${ }^{1}$ The flux units are such that the flux value measured in the V-band is arbitrarily set equal to 1 .

The star-by-star inspection reveals differences between spectrophotometry and photometry which can only be explained by a probable anomaly of the spectrophotometry over all or part of the spectrum of some stars. This type of divergence reappears when we use the description of the passbands given by $R \& M$. The comparison between the $\Phi_{i}(\lambda)$ given by R\&M and those obtained here shows significant differences reaching several percent. In the case of the Paschen continuum $(\lambda>3800$ $\AA)$ these differences are essentially explained by the change in the absolute spectrophotometric reference adopted for $\alpha$ Lyr. The new calibration of the $U$ passband gives a response which is lower by several percent $(\simeq 8)$. The cause of this difference has not yet been interpreted. The application of this new calibration of the $\Phi_{i}(\lambda)$ to the filtering of model atmospheres is not yet finished. As the agreement obtained between models and observations was very satisfactory with the Code version of $\mathrm{R} \& \mathrm{M}$, we could expect a certain discordance when the present functions $\Phi_{i}(\lambda)$ are used. It is still too early to reach conclusions: is there actually a lack of coherence between the models (those of Kurucz) and the photometric observations, or is there a deficiency in the absolute calibration of the G\&S spectrophotometry; or could there be some other unexpected reason? 


\section{PROPOSITION}

The following proposals could lead to the harmonization of the characteristic passbands with the standard colours and magnitudes available on the major photometric systems.

(1) For each standard photometric system, create an inventory of the instrumental passbands and those stars which have both photometric and absolute spectrophotometric data of high accuracy.

(2) Design an efficient method for deriving passband functions consistent with the observed photometric and spectrophotometric data.

(3) Apply this method to provide a coherent collection of all the functions $\Phi_{i}(\lambda)$.

(4) Compare the observed data with synthetic photometry of model atmospheres.

(5) Encourage the responsibility of the observers (particularly in the large observatories) regarding the use of consistent passbands.

\section{REFERENCES}

Gunn, J. E. and Stryker, L. L. 1983, Astrophys. J. Suppl. 52, 121.

Hayes, D. S. and Latham, D. W. 1975, Astrophys. J. 197, 593.

Oke, J. B. and Gunn, J. E. 1983, Astrophys. J. 266, 713.

Rufener, F. and Maeder, A. 1971, Astron. Astrophys. Suppl., 4, 43. 